\title{
Algorithm for recognizing and measuring parameters of biological objects in agriculture based on deep learning convolutional neural networks
}

\author{
Rashid Kurbanov ${ }^{1}$, Nazhmudin Bugaev ${ }^{1,1}$, Alexandr Meshkov ${ }^{1}$, and Stanislav Krivko ${ }^{1}$ \\ ${ }^{1}$ Federal Scientific Agro Engineering Center VIM, 109428, 1st Institutsky proezd, 5, Moscow, Russia
}

\begin{abstract}
As of today existing techniques and tools for measuring leaf area involve the detachment of leaves for further scanning and calculations to determine leaf area. The disadvantages of existing solutions for determining the area of the sheet surface are labor intensity, the duration of these studies, the relatively low accuracy of measurements. Due to these facts this study is an important work aimed to simplifying the process of analyzing biological parameters and other important characteristics of plants, as well as increasing the efficiency of this agrotechnical task. This work aims developing a set of software tools with trained neural networks to determine whether a photographed leaf belongs to the leaves of a soybean crop, assess the health of soybean plants and determine the surface area of a soybean leaf with geotagging.
\end{abstract}

\section{Introduction}

When conducting scientific research related to the methodology for determining the biological potential of plants, a prerequisite for substantiating the prospects of their use in breeding and agricultural practice is the registration of the vegetative state of plants, the determination of the parameters of the assimilation apparatus of the studied plants and the study of the leaf area $[1,2]$.

For breeders and agronomists, the determination of leaf area is a task that must be solved in the course of many quantitative physiological studies of plants. Estimation of leaf area is needed as a morphometric indicator of plant growth. An important characteristic of plants is also the ratio between the area of their leaves and the total mass of the aboveground part, and the height [3].

Determination of the area of the leaf apparatus of plants is necessary for the work of agronomists, breeders, physiologists, biologists, ecologists. In particular, knowledge of the area of the leaf apparatus is needed in agronomy to determine. To date, existing techniques and tools for measuring leaf area imply leaf detachment for further scanning and calculations to determine leaf area [4].

\footnotetext{
${ }^{1}$ Corresponding author: arturnex@gmail.com
} 
Currently, the measurement of leaf area is carried out by weighing paper layouts, by the method of calculating the product of height by width, corrected by the leaf shape factor, or using mechanical planimeters. The applied methods for determining the area of the sheet surface are laborious, cumbersome, associated with the duration of these studies and often cannot provide sufficient measurement accuracy $[5,6,7]$.

In connection with these facts, this study is an important work aimed at simplifying the process of analyzing the biological parameters of plants, as well as increasing the efficiency of this agrotechnical task.

\section{Materials and equipment}

\subsection{Collecting data for a neural network for plant identification}

The collection of data for training the convolutional neural network was carried out on the selection fields in the ISA - a branch of the Federal State Budgetary Scientific Institution FNATS VIM. The amount of training data collected was more than 12,000 images for the main phases of the growing season of soybean plants. This data set is needed to achieve the required recognition accuracy of the soybean leaf apparatus. Data collection was carried out with a Canon EOS 550D camera. Exposure settings, white balance and other settings were selected depending on the shooting conditions. In the future, the obtained materials are subject to processing in specialized software for use in training the algorithm for evaluating the parameters of biological objects and other important characteristics of plants.

\subsection{Development of a software tool for preparing and marking data}

ITS.ImageCroper software was developed to automate the data preparation process. The program allows you to simplify the work of selecting the target segment of the original image (Figure 1). In comparison with existing software solutions, ITS.ImageCroper has a higher speed of selection of the desired segment.

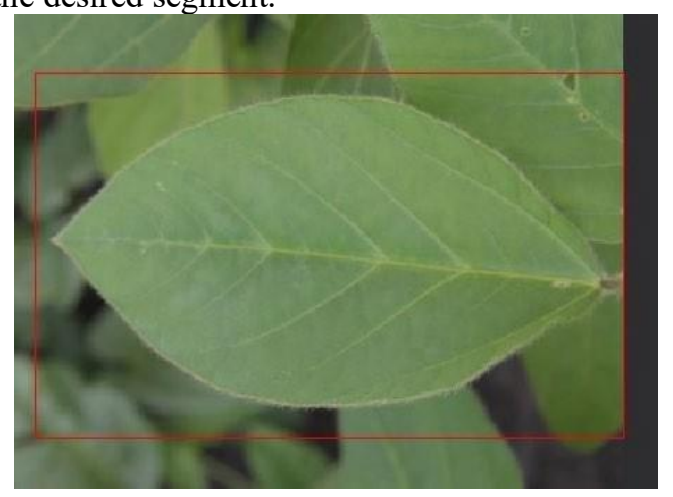

Fig. 1. Select the target segment of the source image.

The software ITS.ImagePrepare was developed, which allows you to automatically create a training image mask from an image cleared from the background.

The program allows you to fully automate the processing of the collected training data for a segmented neural network, automatically create a training image mask from a background-cleared image and a topology of directories and training files for training a segmented neural network. 


\subsection{Development of a software tool for data markup}

The software ITS.ImagePrepare was developed, which allows you to automatically create a training image mask from an image cleared from the background.

The program allows you to:

- fully automate the processing of the collected training data for the neural network segment; automatically create a training image mask from the background cleared image.

- automatically create a topology of directories and training files to train a neural network segment

The appearance of the working software, in the rendering mode of the generated masks, is presented below.

\subsection{Preparing data for training a classification neural network of deep learning}

A test dataset was prepared for training a classification neural network. The entire array of collected data (6000 images) was processed using the ITS.ImageCroper software to select the target segment of the original image. The result is data suitable for training a classification neural network.

\subsection{Data markup for training a deep learning segmented neural network}

A test dataset was prepared for training a segmented neural network. Using the web resource remove.bg, the background data of the array of target image segments was removed (Figure 2). With the help of the ITS.ImagePrepare software, training masks were created from images cleared from the background, and a topology of directories and training files for training a segmented neural network was created.
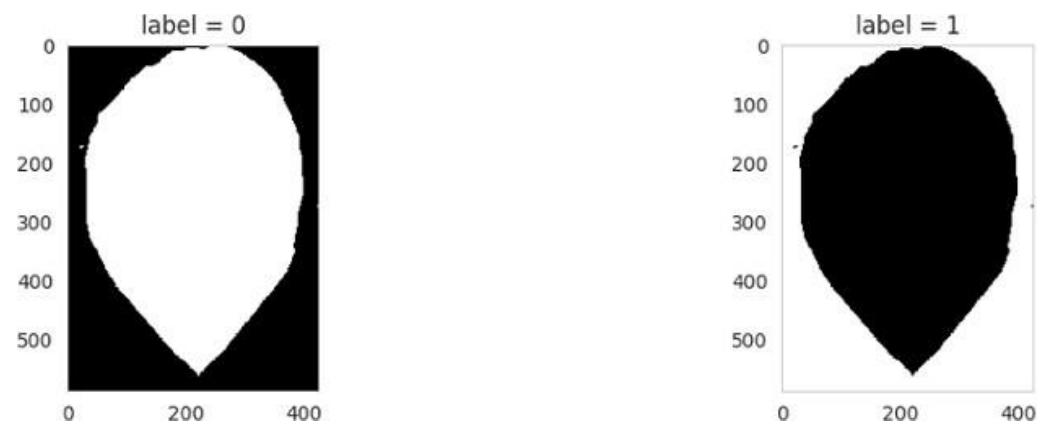

Fig. 2. Selecting the target segment of the original image.

\subsection{Choice of neural network architecture}

A deep learning convolutional neural network must provide functionality and meet the following specifications:

The neural network must evaluate the object presented in the form of a photograph, classify it and give the result in the form of a probability as a percentage of its belonging to a certain biological object with an accuracy of at least $91 \%$ with the volume of training data of at least 6000 images for each class.

Pattern recognition speed should not exceed $0.5 \mathrm{sec}$. one image. 
In case of classification of the presented image as a known biological object, it is necessary to segment the object with an accuracy of at least $85 \%$, the speed of image segmentation should not exceed $0.8 \mathrm{sec}$. one image.

The VGG19 architecture is taken as a basis as the fastest of the satisfying in terms of accuracy. Also, the advantages of the chosen architecture can be attributed to the ability to use the already obtained weights on the classification part for training the segmentation part $[8,9,10,11]$.

\section{Results and discussion}

\subsection{Classification (coding) neural network training}

The architecture of a reconciliation neural network based on the VGG19 architecture for a classification neural network has been developed. The prepared data were divided into training and test data in the ratio of $3 / 4$ and $1 / 4$. The network showed the best results in 5 eras. The number of passes in an epoch is 100 , the size of the training data packet is 32 images.

The following layers have been introduced into the standard CNN VGG19 architecture: $[13,14]$

flatten - an alignment layer of the output data parameters in the vgg 19 network for their processing in subsequent layers.

fc1 - fully connected layer with relu activation function, experience layer. Introducing nonlinearity in network decision making (to reduce the likelihood of memorizing training data). There is a possibility that the network can remember all the training images and show acceptable results based on them, but be very wrong on the new data.

fc2 - Fully connected layer with sigmoid activation function. This layer is used to return the result about the belonging of the input image to a certain class.

During training, the CNN characteristics changed in accordance with the following graphs (Figure 3) on the training data, and after the end of each epoch, the CNN characteristics were checked against the test data (Figure 4).

As a result, we can conclude that the trained CNN shows the required results on new data that the network was not trained with.

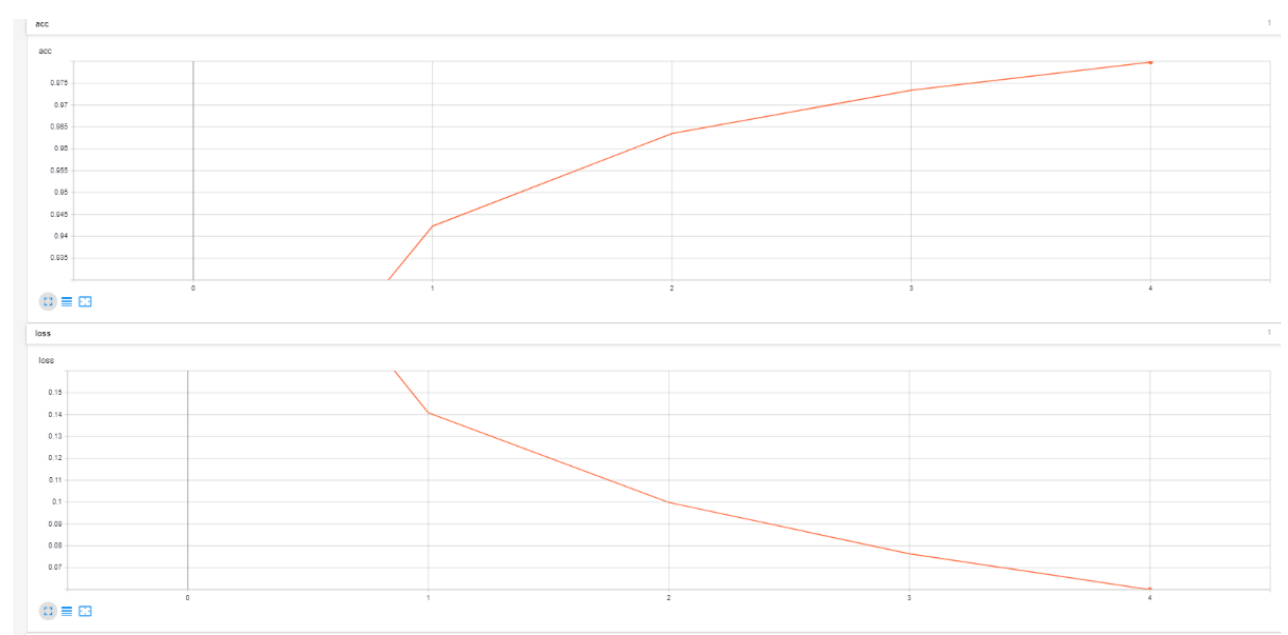

Fig. 3. Plot of accuracy and loss on training data, during training. 


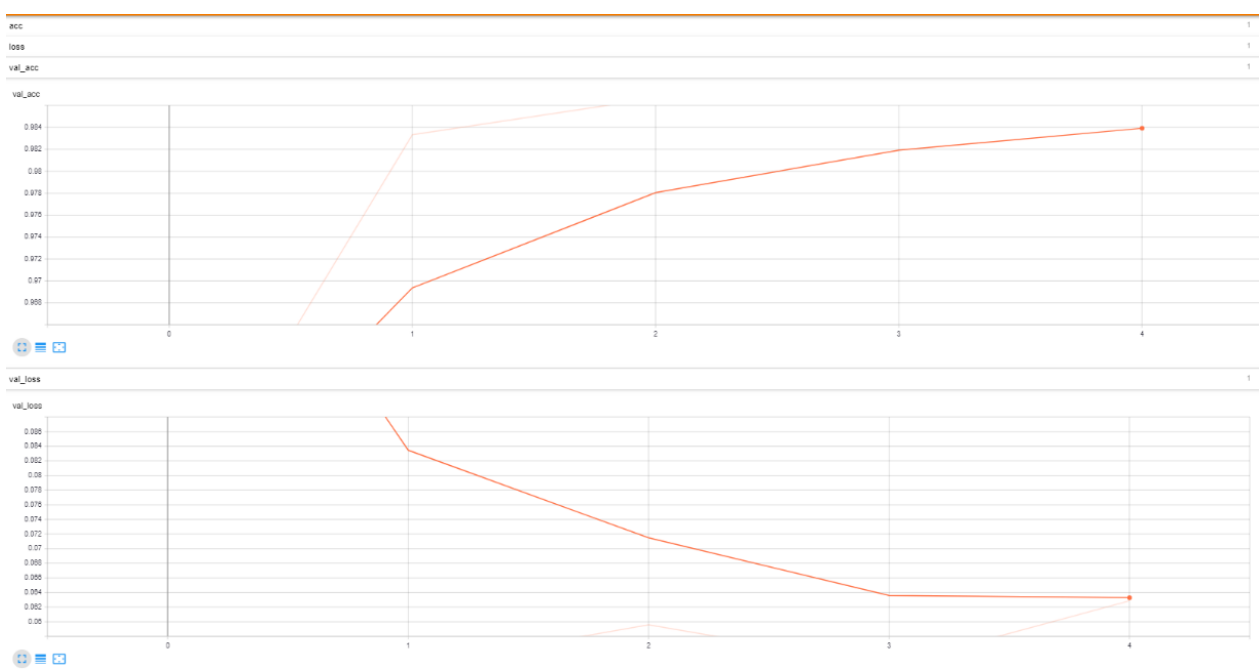

Fig. 4. Accuracy and loss plot on test data during training.

\subsection{Training a segmentation (decoding) neural network}

The architecture of a reconciliation neural network based on the VGG19 architecture for a segmentation neural network has been developed. To train this network, data from the previously trained classification neural network are also used.

The principle of semantic (per-pixel) segmentation was used, with pixel-by-pixel image restoration at the deconvolution stage (restoration of the original image). The prepared data were divided into training and test data in the ratio of $3 / 4$ and $1 / 4$.

The network showed the best results in the 400 era. The number of passes in an epoch is 100 , the size of the training data packet is 30 images, 30 masks. Each image is linked to a corresponding mask.

Into the standard VGG19 neural network were introduced the following layers:

Pool1_11 - fully connected layer with relu activation function, with experience function. Introducing nonlinearity in network decision making (to reduce the likelihood of memorizing training data). There is a possibility that the network can remember all the training images and show valid results based on them, but be very wrong on the new data.

Pool2_11 - fully connected layer similar

Pool1_11. conv2d_transpose - deconvolution layer that doubles the resolution. Pool3_11 - fully connected layer similar

Pool1_11. conv2d_transpose $-4 \mathrm{x}$ deconvolution layer.

Pool4_11 - fully connected layer similar

Pool1_11. conv2d_transpose - deconvolution layer for $8 \mathrm{x}$ resolution. conv6 - fully connected layer similar

Pool1_11, with the function of convolution of the image by 7 times.conv 7 - fully connected layer similar

Pool1_11. conv2d_transpose - deconvolution layer for 16x resolution.

During the training process, the CNN characteristics changed in accordance with the following graphs (Figure 5) on the training data, and after the end of each epoch, the CNN characteristics were checked against the test data (Figure 6). 


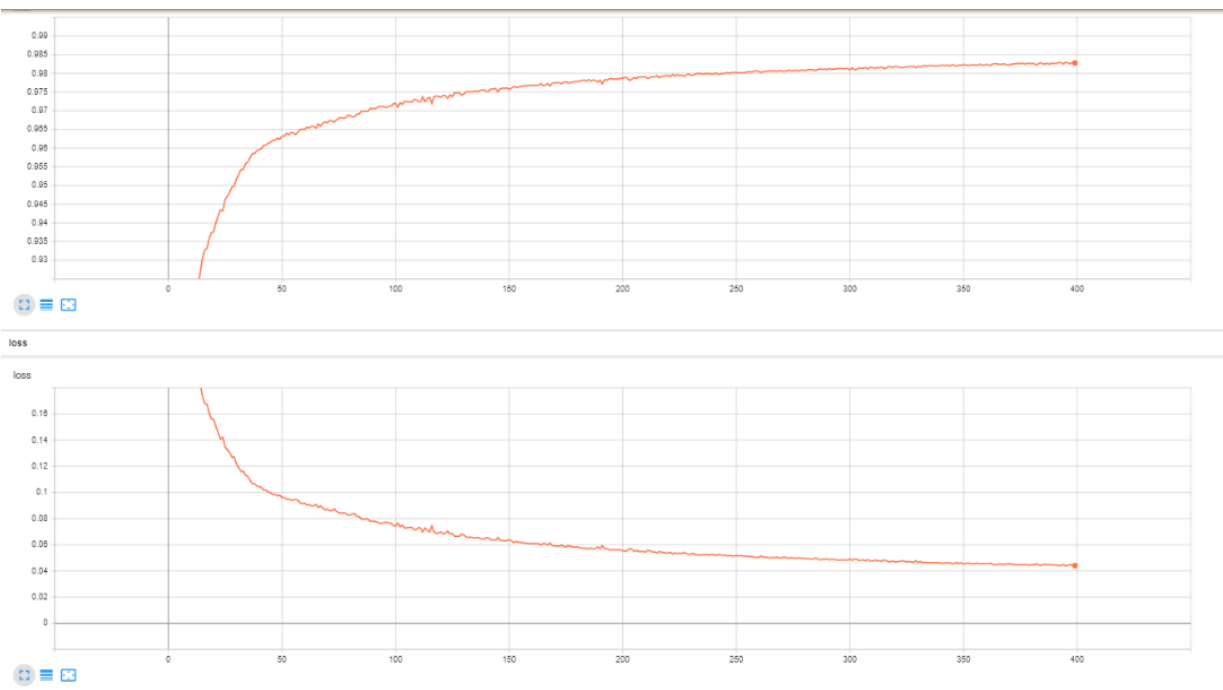

Fig. 5. Graph of accuracy and loss on training data, during training.

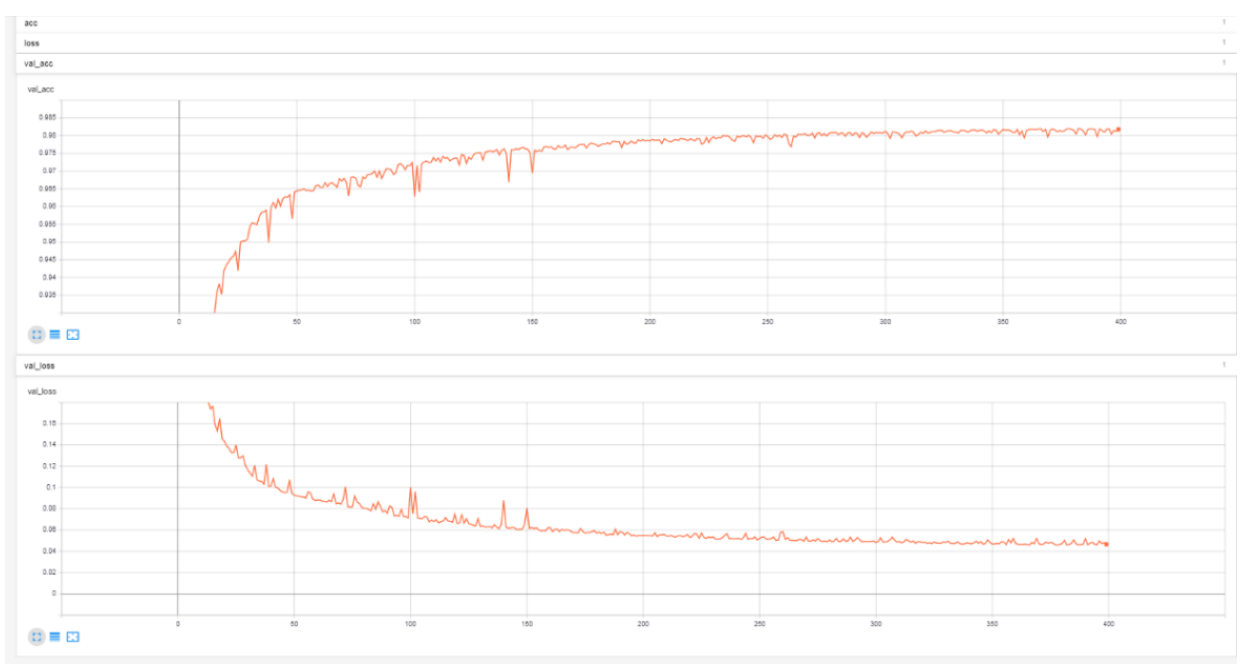

Fig. 6. Accuracy and loss plot on test data during training.

As a result, we can conclude that the trained $\mathrm{CNN}$ shows the required results on new data that the network was not trained with.

\section{Conclusion}

Within the framework of this work

-collected photos of soybean leaves for CNN[15] training;

The volume of training data was 12000 images for the main phases of the growing season of soybean plants. Of these, 6,000 are photographs of healthy leaves and 6,000 of sick leaves. Data collection was carried out in different weather conditions and at different times of the day to achieve higher recognition rates; 
ITS.ImageCroper software tool was developed to automate the data preparation process. It made it possible to speed up the processing of the collected training data and simplified the work on the selection of the target segment of the original image;

he software tool ITS.ImagePrepare was developed, which allows you to automatically create a training image mask for an image cleared from the background. It made it possible to fully automate the processing of the collected training data for a segmentation neural network, automatically create a training image mask from an image cleared from the background, and automatically create a topology of directories and training files;

performed the preparation of a test dataset for training a classification neural network; -performed the markup of the test dataset for training the segmented neural network;

the VGG19 architecture is taken as a basis as the fastest of those satisfying in accuracy. Also, the advantages of the chosen architecture include the ability to use the already obtained weights on the classification part for training the segmentation part;

-developed the architecture of a reconciliation neural network based on the VGG19 architecture for a classification neural network;

-developed the architecture of a reconciliation neural network based on the VGG19 architecture for a segmentation neural network.

As a result of the work carried out, a complex of software tools with trained neural networks was developed to determine whether a photographed leaf belongs to the leaves of a soybean crop, to assess the health of soybean plants and to determine the surface area of a soybean leaf with georeferenced.

\section{References}

1. Taratukhin O. D., Novikova L. Yu., Seferova I. V., Kozlov K. N., Modeling of soybean phenology using artificial neural networks, Biophysics 64, 3, 563-571 (2019)

2. Niazian, M, Niedbala, G, Machine Learning for Plant Breeding and Biotechnology, AGRICULTURE-BASEL 12-16 (2020)

3. Kharchuk O. A., Kirillov A. F., non-Destructive determination of the leaf surface of soybean plants in seasonal dynamics, Eurasian Union of scientists 2-3 (59), 33-36 (2019)

4. Samarkina E. I., Samarkina N. I., Sokolova I. G., Zharov I. N., Method for measuring the parameters of sheet plates using a digital image using specialized software, Plant resources 4(55): 537-547 (2019)

5. Kurbanov R. K., Zakharova O. M., Zakharova N. I., Gorshkov D. M., Software for monitoring and controlling indicators of soybean crop selection processes, Innovations in agriculture 3 (32), 122-132 (2019)

6. Machado B.B., BioLeaf: A professional mobile application to measure foliar damage caused by insect herbivory, Computers and Electronics in Agriculture 129, 44-55 (2016)

7. T. Dauma, H. Buchwaldb, A. Gerlicherb, R. Birnera, Smartphone apps as a new method to collect data on smallholder farming systems in the digital age: A case study from Zambia, Computers and Electronics in Agriculture 153, 144-150 (2018)

8. Tutygin V. S., al Vindi B. H. M. A., Ryabtsev I. A., System for recognizing plant diseases from leaf images based on fuzzy logic and neural networks, Modern science: actual problems of theory and practice. Series: Natural and technical Sciences 3, 107-115 (2019)

9. Sh.P. Mohanty, D.P. Hughes, M. Salathé, Using Deep Learning for Image-Based Plant Disease Detection, Frontiers in Plant Science 7, 14-19

10. P.K. Gikunda, N. Jouandeau, State-of-the-Art Convolutional Neural Networks for Smart Farms: A Review, Advances in Intelligent Systems and Computing 763-775 (2019) 
11. Sala F., Arsene G.G., Iordănescu O., Boldea M., Leaf area constant model in optimizing foliar area measurementin plants: A case study in apple tree, Sci. Hortic. 193, 218-224 (2015)

12. Roginsky A. S., Moshtyls. O., Assessment of the relative area of damage by larvae of the chestnut mining moth (Cameraria ohridella) to the leaves of complex leaves of flowering and non-flowering specimens of the Siberian chestnut in green areas of Minsk. - 75th scientific conference of students and postgraduates of the Belarusian state University 2, 342-346 (2018)

13. A.I. Dyshekov, I.G. Smirnov, M.A. Mirzaev, M.A. Shereuzhev, Published Principles of functioning of the autonomous device for weed control for precision agriculture, Published under licence by IOP (2020)

14. A.I. Dyshekov, I.G. Smirnov, M.A. Shereuzhev, Development of algorithm and technical device for weed recognition, Innovation in agriculture 28, 288-294

15. Sladojevic, Srdjan \& Arsenovic, Marko \& Anderla, Andras \& Stefanović, Darko. Deep Neural Networks Based Recognition of Plant Diseases by Leaf Image Classification. Computational Intelligence and Neuroscience 1-11 (2016)10.1155/2016/3289801. 